\title{
THE RANGE OF $T f$ FOR CERTAIN LINEAR OPERATORS $T$
}

\author{
R. R. PHELPS
}

It is well known that any linear functional $L$ on $C(X)$ (real or com. plex) such that $\|L\|=1=L(1)$ is positive, i.e., $L f \geqq 0$ whenever $f \geqq 0$. More generally, this can be used to show that an operator $T$ from $C(X)$ into $C(Y)$ is positive provided $\|T\|=1$ and $T 1=1$. The positivity of $T$ is equivalent of course, to the assertion that if the range of $f$ is contained in the nonnegative real axis, then so is the range of $T f$. The theorem we prove below says more than this, in that it gives a description of the range of $T f$ in terms of the range of $f$, for arbitrary $f$. Furthermore, the proof is very simple and elementary.

Let $A$ and $B$ be linear spaces of bounded complex-valued functions on the sets $X$ and $Y$, respectively, with the supremum norm. We assume that both $A$ and $B$ contain the constant functions. If $Z$ is a subset of the complex plane, conv $Z$ denotes the closed convex hull of $Z$.

Theorem. Suppose that $T$ is a linear operator from $A$ to $B$. Then $\|T\|=1$ and $T 1=1$ if and only if $(T f)(Y) C \operatorname{conv} f(X)$ for each $f$ in $A$. The operator $T$ is an isometry and $T 1=1$ if and only if $\operatorname{conv}(T f)(Y)$ $=\operatorname{conv} f(X)$ for each $f$ in $A$.

Proof. The key to the proof is the observation that if $K$ is a bounded closed convex set of complex numbers and $\beta \notin K$, then there exists a closed disc $\{z:|z-\alpha| \leqq r\}$ ( $\alpha$ complex, $r>0$ ) which contains $K$ but not $\beta$. Thus, if $Z$ is a bounded subset of the plane, then $\operatorname{conv} Z$ is the intersection of all closed discs which contain $Z$. Suppose, now, that $\|T\|=1, T 1=1$ and $f \in A$. If $f(X)$ is contained in the disc

$$
\{z:|z-\alpha| \leqq r\},
$$

then, for any $y$ in $Y$, we have $r \geqq\|f-\alpha 1\| \geqq\|T(f-\alpha 1)\|=\|T f-\alpha 1\|$ $\geqq|(T f)(y)-\alpha|$, so that $(T f)(y)$ is in the same disc, and hence $(T f)(Y)$ $C$ conv $f(X)$. Conversely, if the latter is true for every $f$ in $A$, then it is immediate that $T 1=1$. Furthermore, for any $f$,

$$
f(X) \subset\{z:|z| \leqq\|f\|\} .
$$

Since $(T f)(Y)$ is in this same disc, $\|T f\| \leqq\|f\|$, and hence $\|T\|=1$. Note that $(T f)(Y) \subset \operatorname{conv} f(X)$ if and only if $\operatorname{conv}(T f)(Y) \subset \operatorname{conv} f(X)$. If we interchange $f$ and $T f$ in the above arguments, we see that (under the hypothesis that $T 1=1$ ) the reverse inclusion is equivalent

Received by the editors December 19, 1963. 
to $\|T f\| \geqq\|f\|$. The assertion about isometries follows immediately from these facts.

Corollary. If $\|T\|=1$ and $T 1=1$, then $T \geqq 0$.

The above proof will yield the same theorem if the functions in $A$ and $B$ have their values in the same real normed linear space $E$, provided every bounded closed convex set in $E$ is the intersection of all the closed balls which contain it. (The condition $T 1=1$ becomes, of course, the condition that $T$ is the identity map on the constant functions in $A$.) Spaces $E$ with this property have been investigated in [1]; they include the Hilbert spaces (finite- and infinite-dimensional) as well as the spaces $l^{p}$ and $L^{p}, 1<p<\infty$. In particular, the above theorem is true for spaces of real-valued functions.

\section{REFERENCE}

1. R. R. Phelps, $A$ representation theorem for bounded convex sets, Proc. Amer. Math. Soc. 11 (1960), 976-983.

UNIVERSITY OF WASHINGTON 